\title{
Nitrotyrosine (NT), a Nitrosative Stress Biomarker, Plasma Concentrations in Gallstone Disease and Cancer Patients
}

\author{
IINA SAIMANEN $^{1}$, DINA RAHKOLA ${ }^{1}$, VIIVI KUOSMANEN ${ }^{1}$, JARI KÄRKKÄINEN ${ }^{1}$, \\ TUOMAS SELANDER $^{2}$, ANU HOLOPAINEN ${ }^{3}$, SAMULI ASPINEN ${ }^{1}$ and MATTI ESKELINEN ${ }^{1}$ \\ ${ }^{1}$ Department of Surgery, Kuopio University Hospital and School of Medicine, \\ University of Eastern Finland, Kuopio, Finland; \\ ${ }^{2}$ Department of Science Service Center, Kuopio University Hospital, Kuopio, Finland; \\ ${ }^{3}$ Department of Clinical Chemistry, Eastern Finland Laboratory Center, Kuopio, Finland
}

\begin{abstract}
Background/Aim: The aim of this study was to assess the plasma concentration of the nitrosative stress biomarker nitrotyrosine (NT) in gallstone disease and cancer patients. Materials and Methods: Initially, 114 patients with symptomatic gallstone disease were randomized into the laparoscopic cholecystectomy $(L C)(n=54)$ and the minicholecystectomy (MC) $(n=60)$ groups. The plasma concentrations of $N T$ were measured just before, immediately after (POPI) and $6 \mathrm{~h}$ after operation (POP2). The cancer patients of this study included ten patients with gastrointestinal cancer and 19 patients with gynecological cancer. Results: There was a statistically significant correlation in the median plasma NT concentrations versus plasma catalase (CAT) concentrations in cholecystectomy patients $(r=0.169$, $p=0.001)$. Interestingly, there was a statistically significant inverse correlation between the individual values of the pain assessed and filed using a 11-point numeric rating scale $8 \mathrm{~h}$ postoperatively $\left(N A D_{8}\right)$ and plasma $N T$ median values in cholecystectomy patients $(r=-0.337, p=0.004)$. Conclusion: Patients with high plasma concentrations of NT appeared to have significantly lower pain scores $8 \mathrm{~h}$ postoperatively.
\end{abstract}

Nitrotyrosine (NT) has been identified as a biomarker of nitrosative stress by reactive nitrogen species (RNS) production. NT is a stable endproduct of nitration of tyrosine by the RNS such as nitrogen dioxide and peroxynitrite $\left(\mathrm{ONOO}^{-}\right)$. The $\mathrm{ONOO}^{-}$is a strong oxidant and toxic due to its ability to oxidize enzymes, lipids, proteins and DNA (1-3).

This article is freely accessible online.

Correspondence to: Matti Eskelinen, MD, School of Medicine, University of Eastern Finland, P.O. Box 100, FI-70029 KYS, Finland. Tel: +358 17173311, Fax: +358 17172611, GSM: +358 400969444, e-mail: matti.eskelinen@kuh.fi

Key Words: Gallstone disease, cancer, surgery, nitrosative stress, plasma nitrotyrosine, pain score.
Since NT is the end product of $\mathrm{ONOO}^{-}$oxidation, assessment of plasma NT concentration could be a useful biomarker of nitrosative stress status in patients who underwent surgery for cholelithiasis. Our original hypothesis was that no difference in oxidative stress (OS) between the laparoscopic cholecystectomy (LC) and minicholecystectomy (MC) procedures would be detected (4). Our results suggest that both LC and MC are safe and feasible operations and have a quite similar short- and long-term outcome $(4,5)$. Interestingly, the LC patients had significantly shorter convalescence and lower pain score at $24 \mathrm{~h}$ post-operatively (6). In addition, we have earlier reported that cholecystectomy patients with high plasma catalase (CAT) concentrations appeared to require significantly fewer analgesic doses during the first $24 \mathrm{~h}$ postoperatively (7). However, the nitrosative stress biomarker NT plasma concentrations in gallstone disease patients versus cancer patients is unknown. Therefore, the present study was designed to investigate the NT plasma concentrations in gallstone disease patients versus cancer patients. Firstly, the main aim of this study was to determine the correlation between plasma NT and CAT levels in patients with benign disease versus patients with cancer and secondly to determine the association between plasma NT concentrations and the individual pain scores postoperatively.

\section{Materials and Methods}

The study was approved by the Ethics Committee of the Kuopio University Hospital District, Kuopio, Finland (DNRO 27/02/2013), registered in the ClinicalTrials.gov database (ClinicalTrials.gov Identifier: NCT01723540, Consort diagram, Figure 1), and was conducted in accordance with the Declaration of Helsinki. The study was a prospective, randomized, multicenter clinical trial consisting of 114 patients with confirmed symptomatic cholelithiasis. Written consent was collected from participants, after receiving verbal and written information about the study. Patients were randomized to undergo either $\mathrm{LC}(\mathrm{n}=54)$ or $\mathrm{MC}(\mathrm{n}=60)$ at two separate hospitals in Finland: Kuopio University Hospital, Kuopio $(n=86)$ and Helsinki University Central Hospital, Helsinki $(n=28)$ 
between March 2013 and May 2015. The design of the study is presented in Figure 1. After patient enrolment, randomization was achieved with a sealed envelope method either to LC or MC groups. Patients with cancer, recruited from our previous study, included 10 patients with gastrointestinal cancer and 19 patients with gynecological cancer (8-10).

The surgical techniques used were standardized for both groups The LC procedure was performed using the four-trocar technique (two $10-\mathrm{mm}$ and two 5-mm trocars) and intra-abdominal pressure was set at $12 \mathrm{mmHg}$. Ultrasonic scissors (Harmonic $\mathrm{ACE}^{\circledR}$, Ethicon Endo-Surgery, Cincinnati, OH, USA) were used in both the MC and LC procedures. The gallbladder was dissected from the liver with ultrasonic scissors. The cystic artery was sealed with ultrasonic scissor and two metal clips were inserted into the cystic duct.

The MC technique is minimally invasive open technique with very short wound; the mean $( \pm \mathrm{SD})$ length of skin incision was 4.8 $( \pm 1.6) \mathrm{cm}$ and the rectus muscle was not cut, but split. A skin incision length more than $7,0 \mathrm{~cm}$ or cutting of the rectus muscle was considered as a conversion to conventional open operation. Incisions were infiltrated with local anesthetic $(20 \mathrm{ml}$ ropivacaine $7.5 \mathrm{mg} / \mathrm{ml})$ at the end of the operation. Both study groups were standardized regarding endotracheal anesthesia and post-operative case including the overall pain filed on an 11-point numeric rating scale (NRS; $0=$ no pain; $10=$ most pain). The study protocol was fully described in our earlier original work (4-7).

EDTA-blood samples were obtained before surgery (PRE), immediately after surgery (POP1) and $6 \mathrm{~h}$ post-operatively (POP2) and centrifuged at $1000 \mathrm{G}(2900 \mathrm{rpm})$ for $15 \mathrm{~min}$. Plasma was separated and stored frozen at $-70^{\circ} \mathrm{C}$ until analyzed. Plasma high sensitivity C-reactive protein (hs-CRP) was analyzed with a Cobas 6000-analyzer (Hitachi, Tokyo, Japan) using the method by Roche Diagnostics (Mannheim, Germany). The plasma CAT assays were performed using enzyme-linked immunosorbent assay (ELISA) Kit (SEC418Hu, Cloud-Clone Corporation, USA). The study protocol of CAT was fully described in our earlier original works $(7,12)$. The plasma NT assays were performed using competitive enzyme immunoassay OxiSelect ${ }^{\mathrm{TM}}$ Nitrotyrosine ELISA Kit (STA-305, Cell Biolabs Inc, USA). The manufacturers intra-assay $\mathrm{CV} \%$ and the inter-assay $\mathrm{CV} \%$ were $5.1 \%$ and $5.8 \%$, respectively.

The data were entered and analyzed with a statistical software program (IBM SPSS Statistics 24.0, IBM, Armonk, NY, USA). Differences in the baseline characteristics between groups were tested by the Fisher's exact test and in the case of continuous data the analysis was performed by independent samples $t$-test. Group differences at three time points were tested by the Mann-Whitney $U$-test and the Wilcoxon signed rank test. The results of the laboratory measurements are presented as median with interquartile range as distributions were right skewed. A two-sided $p$-value of less than 0.05 was considered statistically significant. Spearman's method was used to test for correlation of individual CAT versus NT concentrations (Figure 2) and for NT concentrations versus individual NRS postoperatively (Figure 3 ).

\section{Results}

The perioperative variables and data were quite similar between the study groups. In spite of a slightly higher mean body weight in the LC versus the MC group of patients ( $p=0.057$, Table I),

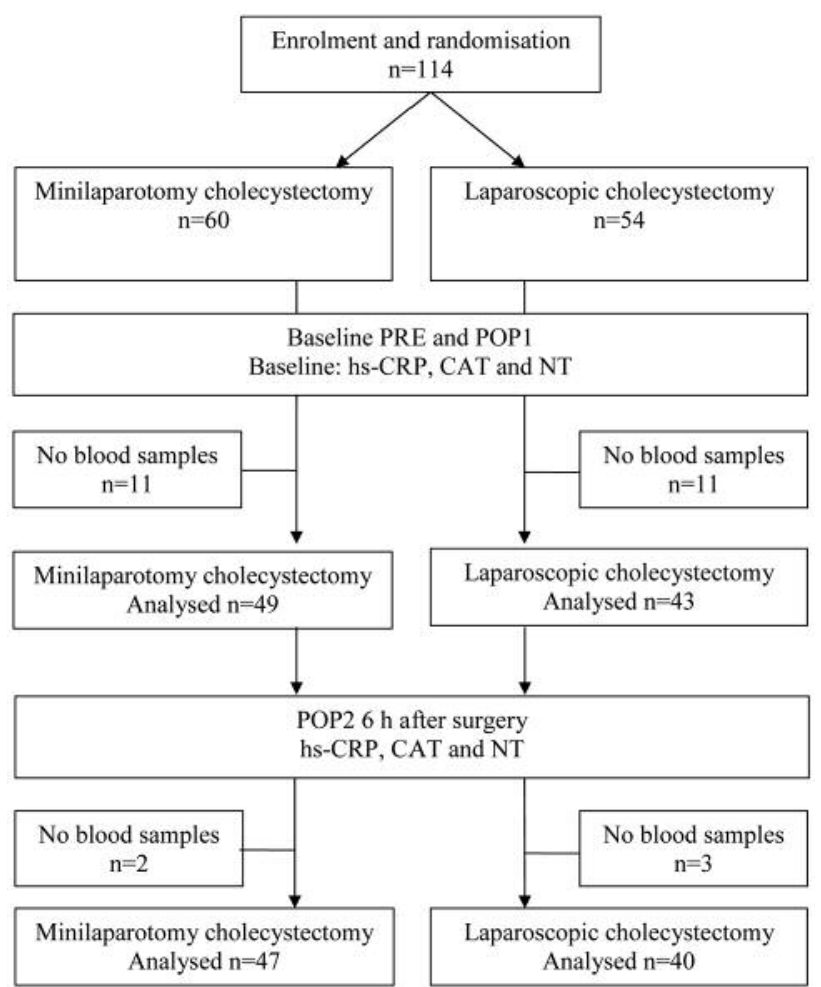

Figure 1. Design of the study as a flowchart. PRE: Sample before surgery; POP1: sample immediately after surgery; POP2: sample $6 \mathrm{~h}$ after surgery; $H s-C R P$ : high sensitivity $C$-reactive protein; CAT: plasma catalase; NT: nitrotyrosine.

there was no statistically significant difference in the mean body mass index (BMI) $(p=0.111$, Table I). Interestingly, the mean length of the skin incision was significantly longer in the LC than in the MC group ( $p<0.001$, Table I).

Table II shows plasma concentrations of hs-CRP, CAT and NT before operation (PRE), immediately after operation (POP1) and $6 \mathrm{~h}$ after operation (POP2) in patients undergoing LC and MC compared to patients with cancer. The median plasma hs-CRP concentrations preoperatively and following surgery in the LC and MC patients were quite similar. Although, cancer patients had a trend for having higher hs-CRP median values preoperatively and following surgery compared to the LC and MC patients, there was no statistically significant difference between the study groups ( $p=0.08, p=0.09, p=0.06$, respectively, Table II). The median plasma CAT concentrations preoperatively and after surgery in the LC and MC patients versus cancer patients were quite similar also and no statistically significant differences were found, although immediately following surgery (POP1) the cancer patients had a trend for having higher CAT levels compared to LC and MC patients (Table II). The median plasma NT concentrations preoperatively and following 


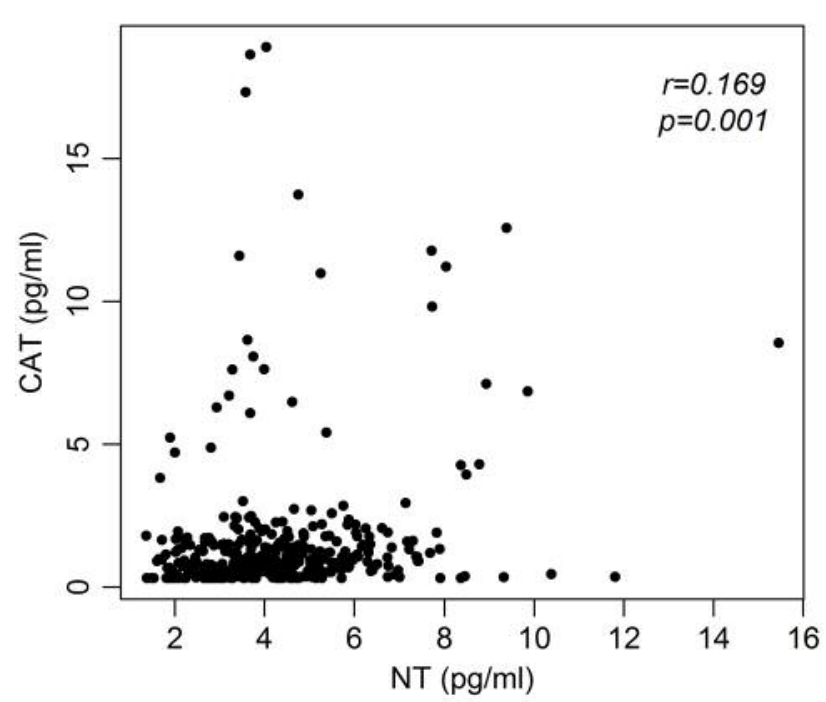

Figure 2. Scatter plots of the plasma nitrotyrosine concentrations (NT) versus plasma catalase (CAT) concentrations in cholecystectomy patients $(r=0.169, p=0.001)$.

surgery in the LC and MC patients versus cancer patients were quite similar and no statistically significant differences were found (Table II).

The postoperative alterations of plasma hs-CRP, CAT and NT marker concentrations in all patients combined are shown in Table III. The median plasma levels of hs-CRP increased by $7.4 \%$ immediately after surgery and this increase was statistically significant $(p=0.021$, Table III). The median plasma CAT concentrations preoperatively and after surgery were quite similar and no statistically significant alterations were found (Table III). The median plasma concentrations of NT slightly decreased by $3.3 \%$ immediately after surgery $(p=0.242$, Table III) and then median plasma concentrations of NT increased $4.4 \% 6 \mathrm{~h}$ following surgery ( $p=0.033$, Table III).

Scatter plots of the plasma NT versus CAT concentrations showed a highly significant correlation between CAT and NT values in cholecystectomy patients $(\mathrm{r}=0.169, p=0.001$, Figure 1$)$.

Figure 3 shows the scatter plots of plasma NT concentrations versus pain assessed using a 11-point numeric rating scale (NRS; $0=$ no pain; $10=$ most pain) at $8 \mathrm{~h}$ postoperatively $\left(\mathrm{NAD}_{8}\right)$. A statistically significant correlation was found between the individual values of the $\mathrm{NAD}_{8}$ and plasma NT median values $(\mathrm{r}=-0.337, p=0.004$, Figure 3 ).

\section{Discussion}

Ter Steege et al. (13) demonstrated immunohistologically the presence of NT in intestinal tissue of celiac disease (CD) patients and concluded that the existence of NT in the plasma

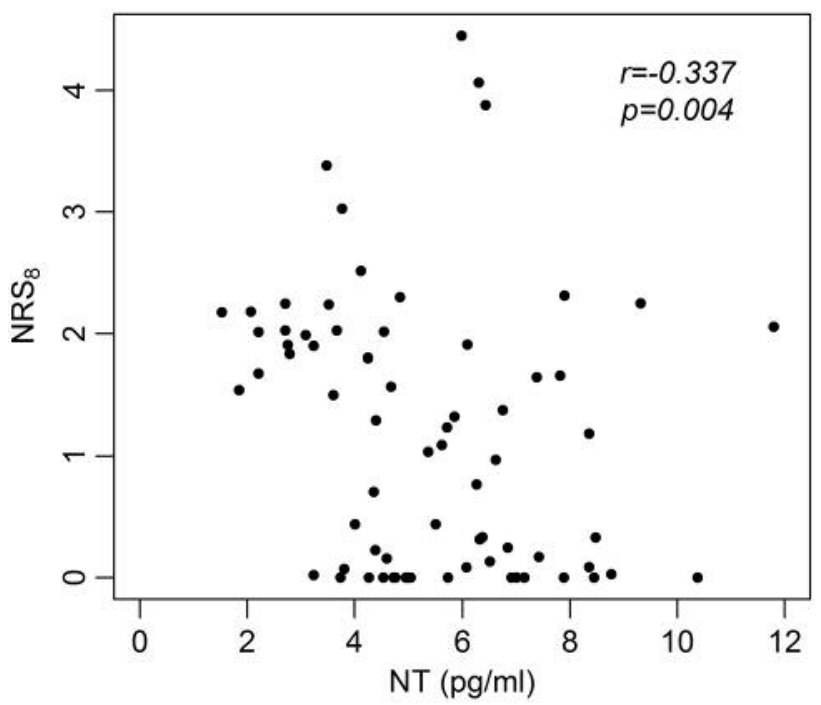

Figure 3. Scatter plots of the plasma nitrotyrosine concentrations (NT) versus pain assessed using a 11-point numeric rating scale (NRS; $0=n o$ pain; $10=$ most pain) at $8 \mathrm{~h}$ postoperatively $\left(N A D_{8}\right)$ in cholecystectomy patients $(r=-0.337, p=0.004)$.

of CD patients could be indirect evidence of the role of NT in the pathophysiology of CD (13). Hintsala et al. (14) investigated the immunohistochemical expression of nitrotyrosine in 36 nevi, 14 lentigo malignant and 71 malignant melanomas. In addition, NT plasma concentrations are in septic shock (15) and in type II diabetic patients (16). NT may also have a role in the development of diabetic complications $(16,17)$ and in the pathogenesis of human corneal diseases (18). Enhanced plasma nitrotyrosine concentrations have been linked to the risk of ischaemic brain injury (19) and earlier research shows that plasma NT levels can be reduced by $\mathrm{N}$-acetylcysteine (20). Eleuteri et al. (21) reported increased NT plasma concentrations in patients with chronic heart failure. In addition, active and passive smoking could decrease plasma NT levels (22). Fiaccadori et al. found that radiocontrast media administration is associated with enhanced plasma NT levels $72 \mathrm{~h}$ following the procedure and this should be taken in consideration in clinical practice, especially, when treating patients with kidney diseases (23).

The assessment of the nitrosative stress biomarker NT plasma concentrations in cholecystectomy patients versus cancer patients is rarely reported. Arsalani-Zadeh et al. (24) and Yiannakopoulou et al. (25) reviewed the literature to compare ROS/RNS in open surgery (OS) and laparoscopic surgery (LS). Sixteen clinical trials investigated the impact of OS and LS on ROS/RNS, however, there were no studies available on plasma NT measurements. In our study, the plasma concentrations of the NT marker were measured just before, immediately after (POP1) and $6 \mathrm{~h}$ after operation (POP2). The first endpoint of this study was to determine the 
Table I. Baseline demographic characteristics and surgical data for the two study groups. Values are mean (standard deviation) or number of cases.

\begin{tabular}{lccc}
\hline Variable & $\begin{array}{c}\text { Minilaparotomy } \\
\mathrm{n}=60\end{array}$ & $\begin{array}{c}\text { Laparoscopy } \\
\mathrm{n}=54\end{array}$ & $p$-Value \\
& & $53.2(13.1)$ & 0.316 \\
Age $($ years $)$ & $11 / 49$ & $18 / 36$ & 0.066 \\
Gender (male/female) & $167.4(7.6)$ & $168.9(9.9)$ & 0.355 \\
Height $(\mathrm{cm})$ & $77.3(14.4)$ & $83.0(17.4)$ & 0.057 \\
Weight $(\mathrm{kg})$ & $27.6(4.4)$ & $29.1(5.6)$ & 0.111 \\
BMI $\left(\mathrm{kg} / \mathrm{m}^{2}\right)$ & $70.0(26.9)$ & $70.1(35.3)$ & 0.976 \\
Operative time $(\mathrm{min})$ & $120.4(28.5)$ & $127.1(35.8)$ & 0.287 \\
Time in the operation theatre $(\mathrm{min})$ & $41(59)$ & $31(39)$ & 0.290 \\
Perioperative bleed $(\mathrm{ml})$ & 3 & 3 & $1.000 *$ \\
Conversion rate $(\mathrm{n})$ & $49.7(12.1)$ & $78.3(22.7)$ & $<0.001$ \\
Length of the scin incision $(\mathrm{s})(\mathrm{mm})$ & & & \\
\hline
\end{tabular}

BMI: Body mass index. $t$-test and $*$ Fisher's exact test were used.

Table II. Plasma concentrations of high-sensitivity C-reactive protein (hs-CRP), catalase (CAT) and nitrotyrosine (NT) before operation (PRE), immediately after operation (POP1) and $6 \mathrm{~h}$ after operation (POP2) in patients undergoing minicholecystectomy (MC) and laparoscopic cholecystectomy $(L C)$ compared to patients with cancer. Values are median (interquartile range).

\begin{tabular}{|c|c|c|c|c|}
\hline Marker & $\mathrm{MC}$ & $\mathrm{LC}$ & Cancer & $p$-Value \\
\hline \multicolumn{5}{|c|}{ Hs-CRP (mg/l) } \\
\hline PRE & $1.87(0.95-3.35)$ & $2.05(0.63-3.98)$ & $4.7(0.9-62)$ & 0.09 \\
\hline POP1 & $1.95(0.88-3.1)$ & $1.95(0.70-3.70)$ & $8.6(0.8-61)$ & 0.08 \\
\hline POP2 & $4.10(2.05-6.35)$ & $2.80(1.28-4.88)$ & $192(104-373)$ & 0.06 \\
\hline \multicolumn{5}{|c|}{ CAT $(\mathrm{pg} / \mathrm{ml})$} \\
\hline PRE & $0.75(0.42-1.36)$ & $1.09(0.56-1.60)$ & $0.73(0.48-1.30)$ & 0.178 \\
\hline POP1 & $0.79(0.48-1.37)$ & $0.93(0.56-1.65)$ & $1.46(1.13-2.36)$ & 0.052 \\
\hline POP2 & $0.76(0.43-1.22)$ & $1.02(0.67-1.58)$ & $1.12(0.68-1.50)$ & 0.376 \\
\hline \multicolumn{5}{|c|}{ NT $(\mathrm{pg} / \mathrm{ml})$} \\
\hline PRE & $3.79(2.88-5.16)$ & $4.39(2.48-5.71)$ & $5.67(4.10-8.10)$ & 0.547 \\
\hline POP1 & $4.11(2.96-5.26)$ & $4.40(2.76-5.76)$ & $5.28(3.41-6.44)$ & 0.925 \\
\hline POP2 & $4.25(3.00-5.45)$ & $4.51(2.96-5.84)$ & $4.69(4.09-6.27)$ & 0.639 \\
\hline
\end{tabular}

Mann-Whitney U-test was used.

RNS stress to surgical trauma measured by plasma NT levels in cholecystectomy patients versus cancer patients. The second endpoint was to determine the correlation between the plasma NT concentration and the individual values of pain, assessed and filed using a 11-point numeric rating scale $8 \mathrm{~h}$ postoperatively $\left(\mathrm{NAD}_{8}\right)$.

In spite of a slightly higher mean body weight in the LC versus the MC patients, we believe that there is no significant bias in the study design, because the difference in the mean BMI is non-significant ( $p=0.111$, Table I). The assessment of the biomarker NT plasma levels in benign disease patients versus cancer patients is rarely reported. However, the median plasma NT concentrations preoperatively and following surgery in the LC and MC patients versus cancer patients were quite similar and no statistically significant differences were found. Therefore, this result did not support our working hypothesis and we are determined to study the association between plasma NT concentrations and the individual pain scores postoperatively.

In this study, the most important finding was a moderate inverse correlation between plasma NT concentrations and pain score (NRS) at $8 \mathrm{~h}$ following surgery. The classical outcome measures evaluated following surgery are usually perioperative course, complications, morbidity, mortality and long-term outcome (post-cholecystectomy symptoms, gallstone recurrence, long-term complications). Therefore, it will be important to have also a biomarker available to monitor patient's outcome following surgery.

Although, there is no major breakthrough in nitrosative biomarkers in cancer, some interesting future perspectives are available. Sasaki et al. (28) examined the risk of cancer by classifying gastric mucosa into 4 categories: chronic gastritis (CG) without Helicobacter pylori (HP), chronic active gastritis (CAG) with HP, chronic metaplasia gastritis 
Table III. The postoperative alteration of plasma hs-CRP, CAT and NT marker concentrations in the group of all patients combined. Plasma concentrations before operation (PRE), immediately after operation (POP1) and $6 \mathrm{~h}$ after operation (POP2). Median (interquartile range) values are shown. The Wilcoxon signed-rank test was used.

\begin{tabular}{lccr}
\hline Marker & All patients & Alteration & $p$-Value \\
\hline Hs-CRP (mg/l) & & & \\
PRE & $1.35(0.49-3.15)$ & & \\
POP1 & $1.45(0.50-3.03)$ & PRE vs. POP1 & $0.021^{\mathrm{a}}$ \\
POP2 & $2.35(0.98-4.65)$ & POP1 vs. POP2 & $<0.001^{\mathrm{b}}$ \\
CAT (pg/ml) & & & \\
PRE & $0.80(0.42-1.58)$ & & \\
POP1 & $0.90(0.52-1.61)$ & PRE $v s$. POP1 & $0.132^{\mathrm{a}}$ \\
POP2 & $0.90(0.56-1.43)$ & POP1 vs. POP2 & $0.741^{\mathrm{b}}$ \\
NT (pg/ml) & & & \\
PRE & $4.21(3.24-5.23)$ & & \\
POP1 & $4.07(3.09-5.10)$ & PRE $v s$. POP1 & $0.242^{\mathrm{a}}$ \\
POP2 & $4.25(3.28-5.27)$ & POP1 vs. POP2 & $0.033^{\mathrm{b}}$ \\
\hline
\end{tabular}

$\mathrm{a}_{v s .}$ PRE; ${ }^{\mathrm{b}} \boldsymbol{v s}$. POP1.

without HP (CGM), and chronic gastritis with atypia without HP (CGA). They analyzed NT levels by ELISA from whole-tissue lysates extracted from nitrogen-frozen material and found enhanced NT levels in the order (from lowest to highest) of CG, CAG, CMG and CGA (overall $p$ value $<0.0001$ ). In addition, Sasaki et al. showed that AC (adenocarcinoma) patients had the highest concentration of NT (28). They suggested that CGA mucosa should be viewed as high-risk mucosa for gastric cancer and the patients with CGA should be evaluated further by gastroscopy and biopsy.

In conclusion, the plasma NT concentrations preoperatively and following surgery in the cholecystectomy patients versus cancer patients were quite similar. Cholecystectomy patients with high plasma levels of NT appeared to have significantly lower pain score following surgery, which may suggest that better ROS balance could have a protective role against postoperative pain.

\section{Conflicts of Interest}

The Authors report no conflicts of interest or financial ties to disclose. The Authors alone are responsible for the content and writing of this article.

\section{Authors' Contributions}

All Authors have met all of the following four criteria: 1 . Substantial contributions to the conception or design of the work or the acquisition, analysis, or interpretation of data for the work, 2. Drafting the work or revising it critically for important intellectual content, 3. Final approval of the version to be published, 4. Agreement to be accountable for all aspects of the work in ensuring that questions related to the accuracy or integrity of any part of the work are appropriately investigated and resolved.

\section{Acknowledgements}

The study was funded by the Heikki, Aino and Aarne Korhonen foundation and the EVO-funding of the Kuopio University Hospital.

\section{References}

1 Luanpitpong $\mathrm{S}$ and Chanvorachote $\mathrm{P}$ : Nitric Oxide and Aggressive Behavior of Lung Cancer Cells. Anticancer Res 35: 4585-4592, 2015. PMID:26254346.

2 Bauer G: Central signaling elements of intercellular reactive oxygen/nitrogen species-dependent induction of apoptosis in malignant cells. Anticancer Res 37: 499-514, 2017. PMID: 28179295, DOI: 10.21873 /anticanres. 11342

3 Bauer G: siRNA-based analysis of the abrogation of the protective function of membrane-associated catalase of tumor cells. Anticancer Res 37: 567-581, 2017. PMID: 28179303, DOI: 10.21873/anticanres.11350

4 Aspinen S, Harju J, Juvonen P, Selander T, Kokki H, Pulkki K and Eskelinen MJ: The plasma 8-OHdG levels and oxidative stress following cholecystectomy: a randomised multicentre study of patients with minilaparotomy cholecystectomy versus laparoscopic cholecystectomy. Scand J Gastroenterol 51: 1507-1511, 2016. PMID: 27435790, DOI: 10.1080/003655 21.2016.1208270

5 Harju J, Aspinen S, Juvonen P, Kokki H and Eskelinen M: Tenyear outcome after minilaparotomy versus laparoscopic cholecystectomy: a prospective randomised trial. Surg Endosc 27: 2512-2516, 2013. PMID: 23344510, DOI: 10.1007/s00464012-2770-x

6 Aspinen S, Harju J, Kinnunen M, Juvonen P, Kokki $\mathrm{H}$ and Eskelinen M: A randomized multicenter study of minilaparotomy cholecystectomy versus laparoscopic cholecystectomy with ultrasonic dissection in both groups. Scand J Gastroenterol 51: 354359, 2016. PMID: 26414236, DOI: 10.3109/00365521.2015. 1091496

7 Saimanen I, Kuosmanen V, Kärkkäinen J, Selander T, Aspinen $\mathrm{S}$, Holopainen A, Rantanen $\mathrm{T}$ and Eskelinen M: Cholecystectomy patients with high plasma level of catalase have significantly lower analgesia requirement: a prospective study of two different cholecystectomy techniques with special reference to patients with cancer. Anticancer Res 38: 54175422, 2018. PMID: 30194197, DOI: 10.21873/anticanres.12872

8 Purdy M, Kärkkäinen J, Kokki M, Anttila M, Aspinen S, Juvonen P, Kokki H, Pulkki K, Rantanen T and Eskelinen M: Does rectus sheath block analgesia alter levels of the oxidative stress biomarker glutathione peroxidase: a randomised trial of patients with cancer and benign disease. Anticancer Res 37: 897902, 2017. PMID: 28179349, DOI: 10.21873/anticanres.11396

9 Kärkkäinen J, Aspinen S, Harju J, Juvonen P, Pulkki K and Eskelinen M: Plasma glutathione peroxidase (GPX1) levels and oxidative stress in gallstone patients operated with two different cholecystectomy techniques: a randomized study with special reference to cancer patients. Anticancer Res 37: 6921-6927, 2017. PMID: 29187474, DOI: 10.21873/anticanres.12156 
10 Kärkkäinen J, Selander T, Purdy M, Juvonen P and Eskelinen M: Patients with increased levels of the oxidative stress biomarker SOD1 appear to have dimished postoperative pain after midline laparotomy: a randomized trial with special reference to postoperative pain score (NRS). Anticancer Res 38: 1003-1008, 2018. PMID: 29374733, DOI: 10.21873 / anticanres. 12315

11 Kärkkäinen J, Saimanen I, Selander T, Aspinen S, Harju J, Juvonen P and Eskelinen M: Gallstone patients with enhanced oxidative stress biomarker SOD1 plasma levels have significantly lower number of postoperative analgesic oxycodone doses: A prospective study with special reference to cancer patients. Anticancer Res 38: 3573-3578, 2018. PMID: 29848712, DOI: 10.21873/anticanres. 12630

12 Saimanen I, Kärkkäinen J, Selander T, Purdy M, Kokki M, Kokki $\mathrm{H}$ and Eskelinen M: Plasma catalase in relation to pain following midline laparotomy: a prospective study of patients with benign diseases and patients with cancer. Anticancer Res 38: 6479-6484, 2018. PMID: 30396975, DOI: 10.21873/ anticanres.13011

13 Ter Steege J, Buurman W, Arends JW and Forget PH: Presence of inducible nitric oxide synthase, nitrotyrosine, CD68 and CD14 in the small intestine in celiac disease. Lab Invest 76: 1-8, 1997. PMID: 9251676

14 Hintsala HR, Jokinen E, Haapasaari KM, Moza M, Ristimäki A, Soini Y, Koivunen J and Karihtala P: Nrf2/Keap1 pathway and expression of oxidative stress lesions 8-hydroxy-2'-deoxyguanosine and nitrotyrosine in melanoma. Anticancer Res 36: 1497-1506, 2016. PMID: 27069125

15 Fukuyama N, Takebayashi Y, Hida M, Ishida H, Ichimori K and Nakazawa $\mathrm{H}$ : Clinical evidence of peroxynitrite formation in chronic renal failure patients with septic shock. Free Radic Biol Med 22: 771-774, 1997. PMID: 9119244

16 Ceriello A, Mercuri F, Quagliaro L, Assaloni R, Motz E, Tonutti L and Taboga C: Detection of nitrotyrosine in the diabetic plasma: evidence of oxidative stress. Diabetologia 44: 834-838, 2001. PMID: 11508267, DOI: 10.1007/s001250100529

17 Pacher P, Beckman JS and Liaudet L: Nitric oxide and peroxynitrite in health and disease. Physiological Reviews 87: 315424, 2007. PMID: 17237348, DOI: 10.1152/ physrev.00029.2006

18 Buddi R, Lin B, Atilano SR, Zorapapel NC, Kenney MC and Brown DJ: Evidence of oxidative stress in human corneal diseases. J Histochem Cytochem 50: 341-351, 2002. PMID: 11850437, DOI: $10.1177 / 002215540205000306$

19 Cuzzocrea S, Mazzon E, Costantino G, Serraino I, Dugo L, Calabrò G, Cucinotta G, De Sarro A and Caputi AP: Beneficial effects of n-acetylcysteine on ischaemic brain injury. British Journal of Pharmacology 130: 1219-1226, 2000. PMID: 10903958, DOI: 10.1038/sj.bjp.0703421

20 Ivanovski O, Szumilak D, Nguyen-Khoa T, Ruellan N, Phan O, Lacour B, Descamps-Latscha B, Drüeke TB and Massy ZA: The antioxidant $\mathrm{N}$-acetylcysteine prevents accelerated atherosclerosis in uremic apolipoprotein E knockout mice. Kidney International 67: 2288-2294, 2005. PMID: 15882270, DOI: 10.1111/j.1523$1755.2005 .00332 . \mathrm{x}$
21 Eleuteri E, Di Stefano A, Ricciardolo FL, Magno F, Gnemmi I, Colombo M, Anzalone R, Cappello F, La Rocca G, Tarro Genta F, Zummo G and Giannuzzi P: Increased nitrotyrosine plasma levels in relation to systemic markers of inflammation and myeloperoxidase in chronic heart failure. Int J Cardiol 135: 386390, 2009. PMID: 19046613, DOI: 10.1016/j.ijcard.2008.11.013

22 Jin H, Webb-Robertson BJ, Peterson ES, Tan R, Bigelow DJ, Scholand MB, Hoidal JR, Pounds JG and Zangar RC: Smoking, COPD, and 3-nitrotyrosine levels of plasma proteins. Environ Health Perspect 119: 1314-1320, 2011. PMID: 21652289, DOI: 10.1289/ehp.1103745

23 Fiaccadori E, Maggiore U, Rotelli C, Giacosa R, Lombardi M, Sagripanti S, Buratti S, Ardissino D and Cabassi A: Plasma and urinary free 3-nitrotyrosine following cardiac angiography procedures with non-ionic radiocontrast media. Nephrol Dial Transplant 19: 865-869, 2004. PMID: 15031342, DOI: $10.1093 / \mathrm{ndt} / \mathrm{gfh} 039$

24 Arsalani-Zadeh R, Ullah S, Khan S and MacFie J: Oxidative stress in laparoscopic versus open abdominal surgery: A systematic review. J Surg Res 169: 59-68, 2011. PMID: 21492871, DOI: $10.1016 /$ j.jss.2011.01.038

25 Yiannakopoulou EC, Nikiteas N, Perrea D and Tsigris C: Effect of laparoscopic surgery on oxidative stress response: systematic review. Surg Laparosc Endosc Percutan Tech 23: 101-108, 2013. PMID: 23579502, DOI: 10.1097/SLE.0b013e3182827b33

26 Teixeira Teixeira D, Fernandes R, Prudêncio C and Vieira M: 3Nitrotyrosine quantification methods: Current concepts and future challenges. Biochimie 125: 1-11, 2016. PMID: 26921794, DOI: $10.1016 /$ j.biochi.2016.02.011

27 Roy E, Patra S, Madhuri R and Sharma PK: Developing electrochemical sensor for point-of-care diagnostics of oxidative stress marker using imprinted bimetallic $\mathrm{Fe} / \mathrm{Pd}$ nanoparticle. Talanta 132: 406-415, 2015. PMID: 25476325, DOI: 10.1016/ j.talanta.2014.09.033

28 Sasaki T, Kuniyasu H, Luo Y, Kitayoshi M, Tanabe E, Kato D, Shinya S, Fujii K, Ohmori $\mathrm{H}$ and Yamashita Y: Increased phosphorylation of AKT in high risk gastric mucosa. Anticancer Res 33: 3295-3300, 2013. PMID: 23898095
Received December 20, 2018

Revised January 9, 2019

Accepted January 10, 2019 